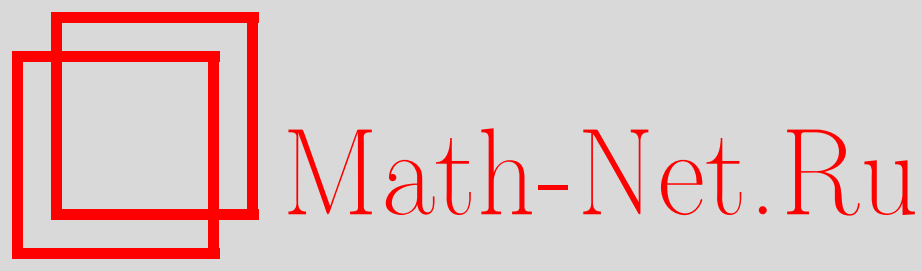

М. И. Клингер, Происхождение бозонного пика и уширения акустических фононов в стеклах, связанное с мягкими модами, ТМФ, 2008, том 154, номер 1, 77-90

DOI: https://doi.org/10.4213/tmf6152

Использование Общероссийского математического портала Math-Net.Ru подразумевает, что вы прочитали и согласны с пользовательским соглашением http://www . mathnet.ru/rus/agreement

Параметры загрузки:

IP: 34.227 .88 .159

26 апреля 2023 г., 16:08:52

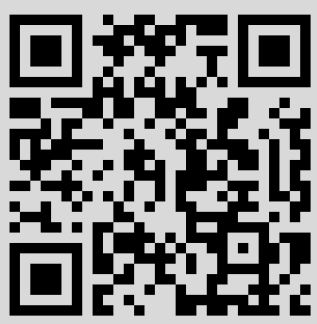




\section{ПРОИСХОЖДЕНИЕ БОЗОННОГО ПИКА И УШИРЕНИЯ АКУСТИЧЕСКИХ ФОНОНОВ В СТЕКЛАХ, СВЯЗАННОЕ С МЯГКИМИ МОДАМИ}

Исследуется происхождение бозонного пика и связанного с ним механизма уширения акустических фононов (ниже пика) в стеклах с высокочастотным звуком выше пика. Происхождение связано с кроссовером Иоффе-Регеля для неупругого (резонансного) рассеяния акустических фононов на колебаниях гармонических мягких мод. Механизм уширения обусловлен резонансным взаимодействием гармонических колебаний мягких мод с акустическими фононами, приводящим к резонансному рассеянию. Найдено, что соответствующая ширина фонона не зависит от температуры и характеризуется степенной частотной зависимостью с показателем, изменяющимся от $\varkappa=2$ непосредственно ниже бозонного пика до $\varkappa=4$ на более низких частотах. Зависимости, по-видимому, частично согласуются с некоторыми недавно полученными экспериментальными данными для рассматриваемых стекол.

Ключевые слова: бозонный пик, динамика стекол, мягкие моды.

\section{1. ВВЕДЕНИЕ}

В данной работе обсуждаются основные свойства мягких мод атомного движения и их влияние на универсальные низкоэнергетические динамические и тепловые свойства неметаллических стекол [1], [2], которые связаны с так называемым бозонным пиком (БП) в спектрах интенсивности неупругого рассеяния фотонов (свет, рентгеновские лучи) и нейтронов при умеренно низких частотных сдвигах $\nu$ (далее, как правило, называемых частотами) [3], [4]. В общем случае происхождение универсальных низкоэнергетических свойств, радикально отличных от свойств кристаллических материалов, принято связывать с возникновением в стеклах дополнительных, недебаевских, возбуждений с низкими энергиями $\varepsilon \ll k T_{\mathrm{D}}=h \nu_{\mathrm{D}}$, много меньшими дебаевской энергии [5], [3]. Имеются существенные различия между свойствами при очень низких частотах $\nu \lesssim \nu_{m} \approx 3 \cdot 10^{-3} \nu_{\mathrm{D}}$ и температурах $T \lesssim T_{m}=h \nu_{m} / k \approx 1 \mathrm{~K}$ (например, изменение удельной теплоемкости $C \propto T$ и теплопроводности $\chi \propto T^{2}$ при возрастании $T$ ) и при умеренно низких частотах

* Department of Physics, Bar-Ilan University, Ramat Gan, Tel Aviv, Israel.

E-mail: klingem@mail.biu.ac.il 
$3 \cdot 10^{-2} \nu_{\mathrm{D}} \lesssim \nu \lesssim 3 \cdot 10^{-1} \nu_{\mathrm{D}}$ и температурах $10 \mathrm{~K} \lesssim T \lesssim 10^{2} \mathrm{~K}$, для которых характерно возникновение БП [3].

Концепция атомных мягких мод и возбуждений с неакустическими мягкими модами при низких энергиях была введена в работе [6], а соответствующая теоретическая модель мягких мод для стекол была впервые развита в 1980-1983 гг. (см. [7]) и в последующие два десятилетия [1], [8], в особенности для теоретического описания универсальных динамических и тепловых свойств стекол при низких частотах $\left(\nu \ll \nu_{\mathrm{D}} \approx 10\right.$ ТГц - дебаевская частота) и низких температурах $\left(T \ll T_{\mathrm{D}} \equiv h \nu_{\mathrm{D}} / k_{\mathrm{B}} \approx 3 \cdot 10^{2} \mathrm{~K}\right)$ и связанных с ними дополнительных возбуждений с очень низкой энергией. Хотя в этой модели предполагается возникновение мягких мод в любом неупорядоченном твердом теле, ожидается, что эти моды будут наиболее многочисленны в стеклах, скорее всего из-за наличия в таких материалах ярко выраженных микроструктур среднего масштаба. При этом общепринятыми являются неколебательная природа возбуждений с очень низкими энергиями $\varepsilon$ и их связь с атомным туннелированием (туннельные состояния) [3], [5], тогда как возбуждения с умеренно низкими энергиями $\varepsilon$, связанные, в частности, с БП, в общем случае оказываются гармоническими колебательными возбуждениями [9]. На самом деле оба типа возбуждений представляются связанными с модами атомного движения одного и того же типа, как подсказывает эмпирическая корреляция [10]: в экспериментах обнаруживается, что универсальные тепловые аномалии, в частности аномалия удельной теплоемкости $C \propto T$, отсутствуют при очень низких температурах в материалах, в которых БП не наблюдается при умеренно низких частотах. Однако единственными модами атомного движения, совместимыми с этой корреляцией, представляются мягкие моды: соответствующие им возбуждения при очень низких энергиях являются неколебательными и связаны с атомным туннелированием, тогда как при умеренно низких энергиях им соответствуют гармонические колебательные возбуждения [1]. В действительности мягкая мода была введена как мода атомного движения, характеризуемая малой эффективной упругой постоянной $k \ll k_{0}$ и, следовательно, низкой эффективной частотой гармонических колебаний $\nu \ll \nu_{\mathrm{D}}$, сосуществующих и взаимодействующих с низкочастотыми акустическими фононами в стеклах. Как и в кристаллических материалах, низкочастотные фононы следует отождествить с дебаевскими фононами, связанными с атомными гармоническими колебаниями подавляющего большинства атомов с упругой постоянной стандартного масштаба $k \approx k_{0}$ (например, для ковалентной или ионной связей $k_{0} \approx 10-20$ эВ $\left./ \AA^{2}\right)$.

Предполагается, что в стеклах, где высокочастотный звук (ВЧЗ) наблюдается выше БП с характерной частотой $\nu_{\text {Бп }} \approx 1$ ТГц, в спектрах неупругого рассеяния фотонов или нейтронов [3], [4] решающую роль в происхождении БП играют колебательные возбуждения мягких мод, или “дефектов" с мягких модами, структуры стекла [1]. В частности, следующие данные недавних экспериментов по рентгеновскому и нейтронному рассеянию в стеклах (см., например, [11]) также можно объяснить в рамках качественной (а скорее всего, верной и по порядку величины) аппроксимации в модели мягких мод (см., например, [2], [12] и ниже разделы 2, 3): 
1. Квазиакустические возбуждения с законом дисперсии $\nu(q) \simeq s|\mathbf{q}|$ наблюдаются при волновых векторах $|\mathbf{q}|$ и частотах $\nu(\mathbf{q})$ вплоть до значений $\nu(\mathbf{q}) / \nu_{\mathrm{D}} \sim 1 / 2$ и $|\mathbf{q}| / q_{\mathrm{D}} \sim 1 / 2$, включая $\nu \approx \nu_{\text {БП }} \ll \nu_{\mathrm{D}}$, при $q_{\mathrm{D}}=\nu_{\mathrm{D}} / s$; эффективная скорость звука $s$

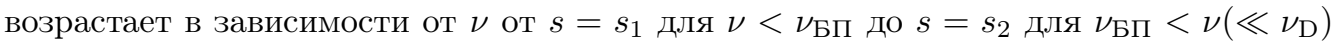
при $0<s_{2}-s_{1} \ll s_{2}$.

2. Ширина этих возбуждений пропорциональна избыточной плотности состояний $\Delta g_{\text {exс }}(\nu)$ дополнительных, недебаевских, гармонических колебаний, $2 \gamma_{\mathrm{ac}}(\nu)$ $\propto \Delta g_{\text {exс }}(\nu)$, причем максимум плотности избыточных состояний характеризует БП.

3. Ширина квазиакустических возбуждений с умеренно низкими частотами не

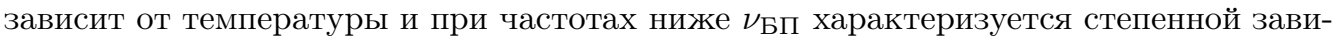
симостью $2 \gamma_{\text {ас }}(\nu) \propto \nu^{\varkappa}$, где, в частности, $\varkappa \approx 2$.

В отличие от универсальных свойств стекол при очень низких частотах и температурах, при умеренно низких частотах и температурах наблюдаются как универсальные (появление БП), так и неуниверсальные свойства (ВЧЗ возникает выше БП в некоторых стеклах, но не появляется в других) [4]. Это объясняется тем [13], что происхождение БП определено общим, слабо зависящим от температуры явлением, называемым кроссовером Иоффе-Регеля (КИР) [14] для акустических фононов или квазиакустических возбуждений от их слабого рассеяния к сильному рассеянию, причем ВЧЗ существует при доминирующем неупругом рассеянии (на гармонических колебаниях мягких мод), но едва ли появляется, когда рассеяние является упругим (на статическом беспорядке). В аналитической теории КИР существует следующая фундаментальная проблема: тогда как слабое рассеяние описывается в рамках теории возмущений разложением по степеням малого параметра $\epsilon=\lambda_{\text {ac }}(\nu) / l_{\text {exc }}(\nu) \ll 1$ или, что эквивалентно, параметра $\epsilon=2 \gamma_{\text {ac }}(\nu) / \nu \ll 1$ для акустических возбуждений (с постоянной скоростью звука) при низких $\nu \ll \nu_{\mathrm{IR}}$, не существует таких разложений при $\epsilon \gtrsim 1$, так что проблема едва ли решается в рамках стандартных квантово-механических подходов при более высоких $\nu \gtrsim \nu_{\mathrm{IR}}\left(\lambda_{\mathrm{ac}}\right.$, $l_{\text {ac }}$ и $2 \gamma_{\text {ac }}$ - соответственно длина волны, средняя длина свободного пробега и ширина акустического возбуждения). Характеристическую частоту $\nu_{\mathrm{IR}} \mathrm{K}$ KР можно найти из общего критерия:

$$
\epsilon\left(\nu_{\mathrm{IR}}\right)=1, \quad \text { например, при } 2 \gamma_{\mathrm{ac}}\left(\nu_{\mathrm{IR}}\right)=\nu_{\mathrm{IR}},
$$

где $\nu_{\mathrm{IR}}$ не зависит от температуры в реальных случаях, когда ширина акустиче-

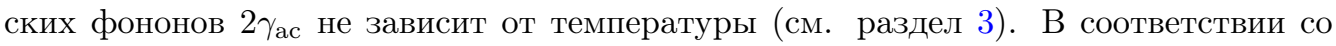
сказанным выше далее рассматриваются такие стекла, в которых БП определяется КИР для неупругого рассеяния на гармонических колебаниях мягких мод при $\nu_{\mathrm{B \Pi}}=\nu_{\mathrm{IR}} \equiv \nu_{\mathrm{IR}}^{(\mathrm{in})}$, а затем следует ВЧЗ при $\nu_{\mathrm{L \Pi}}<\nu \ll \nu_{\mathrm{D}}$.

\section{2. ГАРМОНИЧЕСКИЕ КОЛЕБАТЕЛЬНЫЕ ВОЗБУЖДЕНИЯ}

В этом разделе кратко суммированы некоторые основные результаты теории среднего поля для модели мягких мод динамических и тепловых низкоэнергетических свойств стекол. Для начала можно пренебречь универсальными, относительно слабыми, дипольными (например, упругими) взаимодействиями [15] между мягкими 
модами и рассматривать мягкие моды как независимые (роль эффектов взаимодействия кратко отмечена ниже). Основная концепция модели мягких мод состоит в том, что два типа низкоэнергетических атомных движений и связанных с ними возбуждений, а именно волнообразных акустических фононов низких частот $\nu \ll \nu_{\mathrm{D}}$ и возбуждений мягких мод меньшинства атомов, имеющих относительно низкую концентрацию в локальных конфигурациях, сосуществуют и взаимодействуют друг с другом. Как обычно, низкочастотные акустические фононы больших длин волн $\lambda_{\text {ac }} \gg a_{1} \approx 3 \AA$ (среднее расстояние между атомами) в макроскопическом твердом теле можно аппроксимировать дебаевскими возбуждениями в упругом континууме, пренебрегая деталями атомной структуры. С другой стороны, колебательные возбуждения мягких мод являются резонансными, или квазилокальными, состояниями, подобными некоторым связанным с дефектами колебательным возбуждениям низких частот в кристаллах [16], [17]; обычно в классификации Андерсона [18] эти состояния можно аппроксимировать нелокализованными, или протяженными, хотя они не являются волноподобными, состояниями. Континуальная аппроксимация применима при введении тензора упругих напряжений $e_{i k}$ для характеризации акустических фононов и при описании ответственного за резонансное рассеяние взаимодействия между независимыми "голыми" гармоническими колебательными возбуждениями мягких мод и "голыми" акустическими фононами, оператором $V_{\text {int }}^{(\text {res })}\left(x, e_{i k}\right) \approx \beta_{i k} e_{i k} x$, учитывающим первый неисчезающий член разложения по степеням как смещения мягких мод $x$, так и $e_{i k}$ при реалистичных (безразмерных) значениях $|x|<1$ и $\left|e_{i k}\right| \ll 1 ; \beta_{i k}$ - тензор деформационного потенциала. Пренебрегая для простоты несущественным тензорным характером оператора $V_{\text {int }}^{(\text {(res })}$, мы используем скалярную модель фононов, в которой $V_{\text {int }}^{(\mathrm{res})}\left(x, e_{\mathrm{ac}}\right) \simeq \beta x e_{\mathrm{ac}}[1]$; постоянная скалярного взаимодействия $\beta$ имеет случайный знак и аномально большую

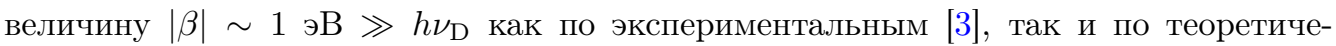
ским [7] оценкам. Это взаимодействие немедленно приводит к не зависящему от температуры резонансному рассеянию при $\varepsilon \simeq h \nu_{\text {ас }}(q)$, в котором или фонон с энергией $h \nu_{\mathrm{ac}}(\mathbf{q})$ поглощается, а возбуждение мягких мод с энергией $\varepsilon$ излучается, или наоборот. Типичный матричный элемент такого взаимодействия можно аппроксимировать следующим образом:

$$
V_{\mathrm{int}}^{(\mathrm{res})}(\varepsilon, q) \approx \boldsymbol{\beta} \cdot(\mathbf{q u}(q)) x(\varepsilon), \quad h \nu_{\mathrm{ac}}(q) \simeq \varepsilon .
$$

Используемый подход модели [2] представляет собой аналитическую теорию типа теории среднего поля, которая аппроксимирует стекло трехмерным изотропным упругим континуумом, содержащим случайно внесенные независимые локализованные "дефекты" - атомные мягкие моды, которые характеризуются частотами гармонических колебаний в диапазоне $\nu^{\prime} \sim 1$ ТГц $\left(\lesssim \nu_{\mathrm{sm}}=\right.$ const $\left.\ll \nu_{\mathrm{D}}\right)$. Теория позволяет вычислять плотность состояний гармонических колебательных возбуждений в неупорядоченной системе, содержащей акустические фононы, взаимодействующие с независимыми осцилляциями гармонических мягких мод. Для последних "голые" гармонические колебательные возбуждения частоты $\nu^{\prime}$ можно характеризовать степенной плотностью состояний, $g_{\mathrm{hv}}\left(\nu^{\prime}\right)=C_{\mathrm{hv}} \nu_{0}^{-1}\left(\nu^{\prime} / \nu_{0}\right)^{2 \alpha}$ с постоянной $C_{\mathrm{hv}} \propto c_{\mathrm{sm}}^{-1}$. Плотность состояний возрастает в зависимости от $\nu^{\prime}$ и содержит два параметра 
материала: типичную частоту мягких мод $\nu_{0} \sim 1$ ТГц, или $\nu_{0} \approx 0.1 \nu_{\mathrm{D}}$, и показатель $\alpha$, меняющийся в диапазоне $2 \leqslant 2 \alpha \leqslant 4$. Обсуждаемая задача подобна задаче из теории колебательных спектров в неупорядоченной трехмерной кристаллической решетке, содержащей конечную атомную концентрацию $c_{\mathrm{d}}$ случайно расположенных дефектов с квазилокальными колебательными возбуждениями; концентрация является низкой в обычном смысле, $c_{\mathrm{d}} \ll 1$, но может быть “высокой” в смысле, указанном ниже (см. также [17]). Тогда данную теорию можно развивать как обобщение подхода для неупорядоченных решеток на континуум с внесенными случайно расположенными мягкими модами. Задача состоит в том, чтобы не только вывести закон дисперсии спектра колебательных частот для данных реализаций конфигурации случайных дефектов, но и найти основные макроскопические спектральные свойства (усредненные по всем реализациям положений случайных дефектов), которые не зависят от случайных микроскопических флуктуаций.

Фундаментальный результат состоит в том, что макроскопические спектральные свойства, такие как основная колебательная плотность состояний $\left.J(\varepsilon)\right|_{\varepsilon=\nu^{2}}=$ $g(\nu) / 2 \nu$ и связанная с ней частотная плотность состояний $g(\nu)$, являются так называемыми самоусредняющимися свойствами, соответствующими макроскопическим характеристикам системы, которые можно вычислить путем усреднения микроскопических характеристик по всем реализациям. Вычисления плотности состояний можно выполнить только для “длинноволновых" акустических фононов, длины волн которых перекрывают большое число (》1) дефектов в мезоскопических подъобъемах, в среднем характеризующихся пространственной однородностью. Область, в которой теория верна, определяется критерием “длинноволновости" акустических возбуждений: типичная длина волны $\lambda_{\mathrm{ac}}\left(\nu_{0}\right)=s_{0} \nu_{0}^{-1} \gg R_{\mathrm{av}}=a_{1} c_{\mathrm{d}}^{-1 / 3}, R_{\mathrm{av}}-$ среднее расстояние между дефектами.

Для часто изучаемых дефектов (например, связанных с тяжелыми изотопами) в кристаллических решетках было явно найдено, что спектры колебательных возбуждений существенно различны в двух различных диапазонах значений $c_{\mathrm{d}}$ : "низкие" концентрации $c_{\mathrm{d}} \ll c_{0}$ и "высокие" концентрации $c_{0} \ll c_{\mathrm{d}} \ll 1$. Характерные значения $c_{0}$ можно определить из уравнения $R_{\mathrm{av}}\left(c_{0}\right)=\lambda_{\mathrm{ac}}\left(\nu_{0}\right)$, так что $c_{0}=\left(\nu_{0} / \nu_{\mathrm{D}}\right)^{3}$, где $\nu_{\mathrm{D}}=s_{0} a_{1}^{-1}, s_{0}$ - средняя скорость звука. Для дефектов с мягкими модами важен только предельный случай "высоких" концентраций, потому что их типичная атомная концентрация равна $c_{\mathrm{sm}} \approx 10^{-2} \gg c_{0} \approx 10^{-3}$ при типичной частоте $\nu_{0} \approx 0.1 \nu_{\mathrm{D}}$. Поскольку "голые" колебательные возбуждения мягких мод характеризуются резонансным взаимодействием с акустическими фононами, неупругое (резонансное) рассеяние акустических фононов на колебательных возбуждениях мягких мод происходит при $\nu_{\text {ac }}(q)=s_{0} q /(2 \pi) \simeq \nu^{\prime}$, где $q$ - величина акустического волнового вектора, $q \ll q_{\mathrm{D}} \equiv 2 \pi \nu_{\mathrm{D}} / s_{0}$. Для развиваемой теории, как и для колебательной динамики в неупорядоченных решетках, основную колебательную плотность состояний $J(\varepsilon)=\pi^{-1} \operatorname{Im} \operatorname{Tr} G_{\mathrm{av}}(\varepsilon)$, как и функцию Грина одиночных возбуждений системы $G_{\mathrm{av}}(\varepsilon)$, можно найти, вычисляя временно́е фурье-преобразование функции Грина $G_{\mathrm{av}}\left(\mathbf{r}-\mathbf{r}^{\prime} ; t\right) \equiv\left\langle G\left(\mathbf{r}, \mathbf{r}^{\prime} ; t\right)\right\rangle$ (для континуальных точек $\mathbf{r}, \mathbf{r}^{\prime}$ ), усредненной по всем реализациям, которая стандартным образом связана с пространственно-временными корреляторами смещений эффективных (усредненных) акустических и мягких мод 
$\left(\mathbf{u}_{\mathrm{ac}}, x_{\mathrm{sm}}\right)$. Уравнения движения для последних, как обычно, можно установить из соответствующего лагранжиана $L=K_{\mathrm{ac}}+K_{\mathrm{sm}}-V_{\mathrm{ac}}-V_{\mathrm{sm}}-V_{\mathrm{ac}-\mathrm{sm}}$, используя стандартные выражения для кинетической $\left(K_{\mathrm{ac}}, K_{\mathrm{sm}}\right)$ и потенциальной $\left(V_{\mathrm{ac}}, V_{\mathrm{sm}}\right)$ энергий акустических и (гармонических) мягких мод и энергии резонансного взаимодействия акустических и мягких мод $\left(V_{\text {int }}^{(\text {res })}\right)$. В актуальном предельном случае "высоких" концентраций $c_{\mathrm{d}}=c_{\mathrm{sm}} \gg c_{0}$ подход на основе функций Грина можно применить для аналитических вычислений плотности состояний $J(\varepsilon)$ в виде степенного ряда по малому параметру $\zeta \equiv R_{\mathrm{av}}^{3}\left(c_{\mathrm{sm}}\right) \lambda_{\mathrm{ac}}^{-3}\left(\nu_{0}\right)=c_{0} / c_{\mathrm{sm}} \ll 1$, в котором основной вклад в вычисляемые величины определяется ненулевым членом низшего порядка. Как обычно, считается, что этот подход хорошо обоснован и последователен в рамках теории возмущений при $c_{0} \ll c_{\mathrm{sm}} \ll 1$, а точность его контролируется малыми поправками (при $\zeta \ll 1)$ к основному члену.

Спектр частот $\nu_{\text {exc }}(q)$ для волнообразных гармонических колебательных возбуждений с волновым вектором $q$, определяющийся резонансными взаимодействиями между акустическими фононами $\left(\nu_{\text {ac }}\right)$ и гармоническими колебаниями мягких мод, можно описывать аналитическим выражением, выведенным из эффективных уравнений движения взаимодействующих мод [2]. Согласно оценке (см. ниже раздел 3) получающиеся возбуждения хорошо определены в том обычном смысле, что их ши-

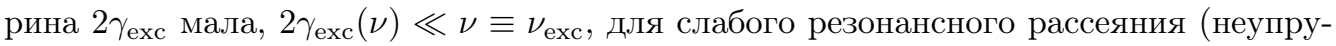
гого рассеяния с малой неупругостью $\Delta \nu \ll \nu)$. Определяющие уравнения рассматриваемой теории содержат как уравнения движения для смещений акустических и мягких мод $\left(\mathbf{u}_{\mathrm{ac}}, x\right)$, так и общее соотношение для фундаментальной колебательной плотности состояний $J(\varepsilon)$. Уравнения движения можно получить, применяя подход функций Грина [17] в адекватной аппроксимации, в которой сумма $\sum_{\mathbf{R}} \beta x(\mathbf{R}) e_{\mathrm{ac}}(\mathbf{R})$ в эффективном гамильтониане, описывающем взаимодействия случайно расположенных $(\mathbf{R})$ мягких мод с акустическими фононами, заменена членом $c_{\mathrm{sm}} \beta x e_{\mathrm{ac}}$, полученным из усреднения суммы по пространственно однородным подобъемам. Тогда уравнения движения имеют (с удержанием основного члена по малому параметру $)$ следующий вид [2]:

$$
\begin{aligned}
\rho \frac{\partial^{2} \mathbf{u}_{\mathrm{ac}}}{\partial t^{2}} & \simeq \rho s_{0}^{2} \nabla_{\mathbf{R}}^{2} \mathbf{u}_{\mathrm{ac}}+c_{\mathrm{sm}} \beta \frac{\partial x_{\mathrm{sm}}}{\partial \mathbf{R}}, \\
\mu_{\mathrm{sm}} \frac{\partial^{2} x_{\mathrm{sm}}}{\partial t^{2}} & \simeq-\mu_{\mathrm{sm}} \nu^{\prime 2} x_{\mathrm{sm}}-\beta e_{\mathrm{ac}}
\end{aligned}
$$

где $\rho$ - средняя массовая плотность упругого континуума. Основную колебательную плотность состояний $J(\varepsilon)$ можно вычислить, используя общую формулу (см. ниже (5)) и спектр собственных значений колебательных возбуждений, следующий из уравнений движения.

Колебательный спектр получается стандартным способом из уравнений (3) только для хорошо определенных волнообразных возбуждений $\left\{x_{\mathrm{sm}}(\mathbf{r}, t), \mathbf{u}_{\mathrm{ac}}(\mathbf{r}, t)\right\} \propto$ $e^{i(\mathbf{q r}-2 \pi \nu t)}$, с волновыми векторами q и малыми ширинами частот $2 \gamma_{\text {exс }} \ll \nu_{\text {exc }}[2]$. Спектр имеет поляритонный характер с двумя ветвями и с законом дисперсии $\varepsilon\left(q, \varepsilon^{\prime}\right)=\epsilon_{j}\left(q, \varepsilon^{\prime}\right) \equiv \nu_{j}^{2}\left(q, \varepsilon^{\prime}\right), j=1,2$, при заданном значении параметра "голых" 
мягких мод $\varepsilon^{\prime} \equiv \nu^{\prime 2}$ :

$$
\begin{aligned}
\varepsilon\left(q ; \varepsilon^{\prime}\right) & =\epsilon_{j}\left(q, \varepsilon^{\prime}\right)= \\
& =\frac{1}{2}\left(\varepsilon^{\prime}+\frac{s^{2} q^{2}}{4 \pi^{2}}+\Delta\right)\left\{1+(-1)^{j}\left[1-\pi^{-2} \varepsilon^{\prime} s^{2} q^{2}\left(\varepsilon^{\prime}+\frac{s^{2} q^{2}}{4 \pi^{2}}+\Delta\right)^{-2}\right]^{1 / 2}\right\},
\end{aligned}
$$

где $q \equiv|\mathbf{q}|=2 \pi / \lambda_{\mathrm{ac}}, \Delta \simeq c_{\mathrm{sm}} Q^{2} \varepsilon^{\prime}, Q^{2}=\beta^{2}\left(\mu_{\mathrm{av}} s_{0}^{2} \mu_{\mathrm{sm}} \nu_{0}^{2} a_{0}^{2}\right)^{-1} \approx 10$ для типичного значения параметра взаимодействия мягких мод $\beta \approx 1$ эВ и $\mu_{\mathrm{av}} s_{0}^{2} \approx 10$ эВ, $\mu_{\mathrm{sm}} \nu_{0}^{2} a_{0}^{2} \sim$ 0.01 эВ $a_{0}=1 \AA$. Ветви разделены узкой щелью ширины $\Delta=\epsilon_{2, \min }-\epsilon_{1, \max } \approx 0.1 \varepsilon_{0}$, где $\epsilon_{2, \min }=\varepsilon^{\prime} \approx \varepsilon_{0} \equiv \nu_{0}^{2}, \epsilon_{1, \max }=\varepsilon^{\prime}\left(1-c_{\mathrm{sm}} Q^{2} / \varepsilon_{0}\right)^{1 / 2}$. В этом состоит наиболее замечательное свойство выведенного спектра возбуждений как гибридизированных акустических и колебательных мягких мод. Если параметр взаимодействия акустических и мягких мод $\beta=0$, щель исчезает $(\Delta=0)$ и возникают две отдельные ветви для невзаимодействующих колебательных возбуждений мягких мод $\nu=\nu^{\prime}$ и акустических фононов $\nu \simeq s_{0} q /(2 \pi)$. При частотах, относительно далеких от щели, дисперсионные соотношения $\nu_{j}^{2}\left(q, \varepsilon^{\prime}\right)$ хорошо определенных возбуждений в действительности весьма схожи с дисперсионными соотношениями акустических возбуждений со скоростью звука $s=s_{1} \simeq s_{0}\left(1-\Delta \nu_{0}^{-2}\right)^{1 / 2}$ и $\nu_{1}^{2}\left(q, \varepsilon^{\prime}\right) \simeq s_{1}^{2} q^{2} /\left(4 \pi^{2}\right)$ при $q<q_{0}=2 \pi \nu_{0} / s_{0}$, тогда как $\nu_{2}^{2}\left(q, \varepsilon^{\prime}\right) \approx \nu_{0}^{2}+s_{2}^{2} q^{2} /\left(4 \pi^{2}\right)$ при $q_{0}<q<q_{\mathrm{M}}=\nu_{\mathrm{M}} / s_{0}$ с большей скоростью звука $s_{2} \simeq s_{0}>s_{1}, s_{2}-s_{1} \ll s_{1}$, в соответствии с экспериментальными данными 1 из раздела 1. Оба типа колебательных возбуждений достаточно далеко от щели имеют малую частотную ширину благодаря слабому резонансному рассеянию мягких и акустических мод, $2 \gamma_{\mathrm{exc}}^{(1,2)} \simeq 2 \gamma_{\mathrm{ac}}^{(1,2)} \ll \nu_{\mathrm{ac}}^{(1,2)}$. Более того, акустическая часть верхней ветви подобна возбуждениям ВЧЗ с эмпириче-

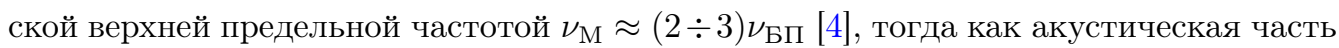
нижней ветви соответствует стандартным дебаевским фононам.

Другая основная черта получающегося спектра колебательных возбуждений гибридизированных акустических и мягких мод состоит в появлении совсем другого типа состояний в диапазоне частот $\sim$ ТГц вокруг щели, которые не описываются волнообразными возбуждениями (4). Не следует ожидать, что эти состояния будут хорошо определенными возбуждениями: их ширины становятся большими, порядка частот, соответствующих КИР (уравнение (1)). Возникновение таких “плохо определенных" возбуждений можно понять из экстраполяции оценки масштаба фононной ширины $2 \gamma_{\text {ac }}(\nu)$ (см. ниже $\left.(12)-(15)\right)$ при $s_{0} q \ll \nu_{0}$ на состояния вокруг краев щели, т.е. от $2 \gamma_{\text {ac }}(\nu) \approx \nu_{0}\left(\nu / \nu_{0}\right)^{\varkappa} \ll \nu_{0}$ при $\varkappa=$ const к $2 \gamma_{\text {ac }}(\nu) \approx \nu$ при $\nu \approx \nu_{\mathrm{IR}}^{\text {(in })} \approx \nu_{0}$, где характеристическая частота $\nu_{\mathrm{IR}}^{(\mathrm{in})}$ отделяет кроссовер от слабого неупругого рассеяния и гибридизации обоих типов “голых" нелокализованных возбуждений к их сильным рассеянию и гибридизации в плохо определенных и, вероятно, нелокализованных возбуждениях.

Спектральной характеристикой, вычисленной в настоящей модели, является основная колебательная плотность состояний $J(\varepsilon)$ для спектра собственных значений колебательных возбуждений при $\varepsilon=\varepsilon(q)=\nu^{2}(q)$ :

$$
J(\varepsilon)=\int d \varepsilon^{\prime} J_{\mathrm{sm}}\left(\varepsilon^{\prime}\right) I\left(\varepsilon ; \varepsilon^{\prime}\right)=\frac{g(\nu)}{2 \nu},
$$


где $J_{\mathrm{sm}}\left(\varepsilon^{\prime} \equiv \nu^{\prime 2}\right)$ - фундаментальная плотность состояний колебательных мягких мод, $I\left(\varepsilon ; \varepsilon^{\prime}\right)$ - ядро, преобразующее "голые" колебательные возбуждения мягких мод в получающиеся колебательные возбуждения благодаря взаимодействию акустических и мягких мод, причем как $J(\varepsilon)$, так и $J_{\mathrm{sm}}\left(\varepsilon^{\prime}\right)$ нормированы одинаково,

$$
\int J(\varepsilon) d \varepsilon=\int g\left(\varepsilon^{1 / 2}\right) d\left(\varepsilon^{1 / 2}\right)=1=\int g_{\mathrm{hv}}\left(\nu^{\prime}\right) d \nu^{\prime} .
$$

Кроме того, для независимых колебаний мягких мод имеем $J_{\mathrm{sm}}\left(\varepsilon^{\prime}\right)=g_{\mathrm{hv}}\left(\nu^{\prime}\right) /\left(2 \nu^{\prime}\right)$ при $g_{\mathrm{hv}}\left(\nu^{\prime}\right) \propto \nu_{0}^{-1}\left(\nu^{\prime} / \nu_{0}\right)^{\alpha}$ и $2 \leqslant \alpha \leqslant 4$. Для акустической части нижней ветви $\nu_{1}\left(q, \varepsilon^{\prime}\right) \simeq s_{1} q$ для возбуждений верна стандартная аппроксимация [12]

$$
I\left(\varepsilon ; \varepsilon^{\prime}\right) \simeq I_{0}\left(\varepsilon ; \varepsilon^{\prime}\right) \propto \int d q q^{2} \delta\left[\varepsilon-\varepsilon\left(q ; \varepsilon^{\prime}\right)\right]
$$

где дельта-функция $\delta(x)=\{0, x \neq 0 ; \infty, x=0\}$. Однако в настоящий момент нет последовательной аналитической теории колебательных состояний и их плотности состояний вокруг КИР или щели. Причина состоит в том, что стандартные разложения теории возмущений в теории рассеяния с малым параметром $\vartheta \equiv$ $\gamma_{\text {ас }}\left(\nu^{\prime}\right) / \nu^{\prime} \ll 1$ не пригодны вокруг КИР при $\vartheta \approx 1$, т.е. при $\varepsilon \approx \nu_{0}^{2}$. Поэтому в данном случае можно производить численные вычисления, в которых дельта-функция в выражении для $I_{0}\left(\varepsilon ; \varepsilon^{\prime}\right)$ аппроксимируется “допредельной” регулярной функцией $\left.D\left[\varepsilon-\epsilon_{j}\left(q ; \varepsilon^{\prime}\right) ; \eta\right]\right|_{\eta=\eta_{1} \ll 1}$, в частности функцией Лоренца, в которой вместо стандартного малого параметра $\eta_{1} \rightarrow 0$ подставлен значительно больший параметр $\eta_{2} \approx 1$. Этот феноменологический анзац соответствует большой ширине возбуждений $2 \gamma_{\mathrm{ac}}^{(\mathrm{in})}\left(\nu_{\mathrm{IR}}^{(\mathrm{in})}\right) \equiv \eta_{2} \nu_{0} \approx \nu_{\mathrm{IR}}^{(\mathrm{in})} \approx \nu_{0}$, характеризующих КИР вокруг спектральной щели:

$$
D\left[\varepsilon-\epsilon_{j}\left(q ; \varepsilon^{\prime}\right) ; \eta_{2}\right]=\frac{\eta_{2} \nu_{0}^{2}}{\pi\left[\left(\varepsilon-\epsilon_{j}\left(q ; \varepsilon^{\prime}\right)\right)^{2}+\eta_{2}^{2} \nu_{0}^{4}\right]}, \quad j=1,2 .
$$

Тогда как вычисленная основная плотность состояний $J(\varepsilon)=g(\nu) /(2 \nu)$, так и редуцированная частотная плотность состояний $g(\nu) / \nu^{2}$ [12], [13] демонстрируют выраженный широкий пик асимметричной формы и большой ширины. Этот пик можно отождествить с БП при $\nu \simeq \nu_{\text {БП }} \simeq \nu_{\mathrm{IR}}^{(\mathrm{in})} \approx \nu_{0}$ с шириной $2 \gamma_{\text {БП }} \simeq 2 \gamma_{\mathrm{IR}}^{(\mathrm{in})} \approx \nu_{\text {БП }}$. Выше БП как $J(\varepsilon)$, так и $g(\nu) / \nu^{2}$ демонстрируют дебаевское поведение, что может также соответствовать ВЧЗ. Можно полагать по определению КИР, что фазовая когерентность акустических фононов существенным образом нарушается в соответствующих плохо определенных возбуждениях, приближенно описываемых суперпозициями акустических волн и квазилокальных колебательных состояний мягких мод и их коэффициентами с нерегулярными фазами, которые нельзя вычислить аналитически в отсутствие последовательного теоретического подхода. Поскольку по определению частота КИР ниже, чем частота "края подвижности", можно полагать для оценок, что собственные состояния в области КИР могут быть скорее нелокализованными, плохо определенными, характеризующимися диффузионным движением и эффективным коэффициентом диффузии $D_{\text {eff }} \approx V_{\text {eff }}^{2} \gamma_{\text {eff }}^{-1}$, чем локализованными состояниями с $V_{\text {eff }}=0$ или волнообразными состояниями со скоростью звука $s_{0}$. Кроме того, групповая скорость $V_{\text {eff }}$ много меньше, чем $s_{0}$ : 
$V_{\mathrm{eff}} \approx\left(a_{1}^{2} \nu_{\mathrm{eff}} \gamma_{\mathrm{eff}}\right)^{1 / 2} \lesssim a_{1} \nu_{\text {БП }} \approx 0.1 s_{0}$ при эффективных частоте и ширине соответ-

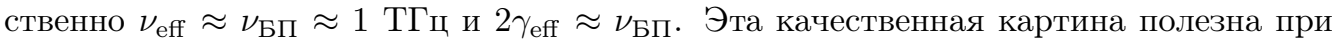
объяснении природы “плато" в теплопроводности рассматриваемых стекол [19], [13].

Перечисленные выше результаты качественно и по порядку величины схожи как в случае, когда универсальные дипольные взаимодействия между “дефектами” мягких мод в структуре стекла не учитываются [2], [12], так и в случае, когда взаимодействия учитываются [13]. При этом модель мягких мод может дать качественное, а вероятно, и по порядку величины, объяснение происхождения БП и экспериментальных данных 1 из раздела 1 для рассматриваемых стекол за счет взаимодействия гармонических колебаний мягких мод с акустическими фононами и наличия КИР для неупругого рассеяния фононов. Далее мы обсудим, как можно объяснить некоторые экспериментальные данные 2, 3 из раздела 1, касающиеся отсутствия зависимости от температуры ширины фононов $2 \gamma_{\text {ac }}(\nu)$, ее степенной зависимости от частоты и соотношения, связывающего ширину фононов с колебательной плотностью состояний мягких мод при низких частотах $\nu<\nu_{\text {БП }}$

\section{3. ШИРИНА АКУСТИЧЕСКИХ ФОНОНОВ: СООТНОШЕНИЯ И ОЦЕНКИ}

Применяя золотое правило Ферми к матричному элементу взаимодействия в формуле (2), можно стандартным способом получить не зависящую от температуры ширину низкочастотных акустических фононов $\left(\nu<\nu_{\text {Бп }}\right)$ :

$$
2 h \gamma_{\mathrm{ac}}(\nu)=2 \pi \sum_{\varepsilon} \sum_{\mathbf{q}}\left|V_{\mathrm{int}}^{(\mathrm{res})}(\varepsilon ; q)\right|^{2} g_{h \nu}(\varepsilon) \delta(\varepsilon-h \nu) \delta\left(\nu-s_{1} q\right),
$$

где $g_{h \nu}(\varepsilon)$ - одночастичная плотность состояний гармонических колебательных возбуждений мягких мод с энергией $\varepsilon$. Преобразуем суммы по непрерывным энергиям $\varepsilon$ и волновым векторам q в интегралы, которые затем можно вычислить; результатом является следующее не зависящее от температуры выражение, которое может в качественном приближении (вероятно, и по порядку величины) объяснить некоторые недавние экспериментальные данные 3 из раздела 1:

$$
2 h \gamma_{\mathrm{ac}}(\nu) \approx C w\left(g_{h \nu}(h \nu) w\right)
$$

где $C=Q_{0}\left(\beta^{2} /\left(M s_{1}^{2} w\right)\right)$, а $M-$ средняя атомная масса. Безразмерный параметр среды $Q_{0}$ можно оценить из сравнения теоретического результата $c_{\mathrm{TS}} \approx Q_{0} h \nu_{\mathrm{m}} / w$, атомной концентрации чрезвычайно низкоэнергетических возбуждений мягких мод (туннельных состояний) с эмпирическим значением $c_{\mathrm{TS}} \approx 10^{-5}$ [5], [3] [1], в результате чего получается $Q_{0} \approx 10^{-4}$ при реалистичных значениях $h \nu_{\mathrm{m}} / w \approx 0.1$. Энергия $w$ является общим энергетическим масштабом мягких мод, $w \sim 10^{-3}$ эВ, который существенно меньше, чем типичная энергия гармонических мягких мод $h \nu_{0} \approx 0.1 h \nu_{\mathrm{D}}$, т.е. $w \ll h \nu_{0} \ll h \nu_{\mathrm{D}}$. При этом оценка соответствующей обратной длины свободного пробега низкочастотных акустических фононов приводит к выражению

$$
l_{\mathrm{ac}}^{-1}(\nu)=2 \gamma_{\mathrm{ac}}(\nu) / s_{1} \approx C\left(w / h s_{1}\right)\left(g_{h \nu}(h \nu) w\right),
$$


которое подобно выражению, полученному другим способом [20], если $g_{h \nu}(h \nu) \propto$

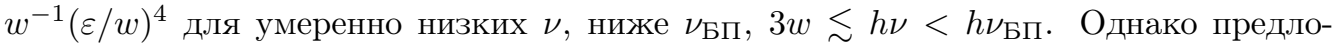
женная конкретная степенная функция, описывающая плотность состояний, не выглядит как общее выражение даже в простейшем случае независимых мягких мод, когда универсальными дипольными взаимодействиями между “дефектами" пренебрегают.

Действительно, по определению (см. раздел 1) мягкая мода характеризуется малой упругой постоянной $k \ll k_{0} \approx M s_{1}^{2} / a_{0}^{2}$ и описывается ангармоническим потенциалом $V(x) \approx M s_{1}^{2}\left(\eta x^{2}+\xi x^{3}+x^{4}\right)$, где случайные безразмерные параметры малы по абсолютной величине, $|\eta| \ll 1$ и $\xi^{2} \ll 1$ [1]. Параметры мягких мод подчинены плотности распределения $F(\eta, \xi)$, определяющей макроскопические свойства:

$$
\bar{A}=\iint d \eta d \xi F(\eta, \xi) A\left(\eta, \xi^{2}\right) .
$$

В общем случае имеются два предельных типа функций $F=F_{\alpha}(\eta, \xi), \alpha=1,2$, в зависимости от характера конкретных межатомных сил в стекле при $\eta \rightarrow 0$ : $F_{1} \approx F_{1}^{0}=$ const $\neq 0$ или $F_{2} \approx|\eta| F_{2}^{0}, F_{2}^{0}=$ const $\neq 0$. Колебательную плотность состояний мягких мод $g_{h \nu}(\varepsilon)$ можно вычислить, применяя стандартное выражение (см., например, [17], [1])

$$
g_{h \nu}^{(\alpha)}(\varepsilon)=\iint_{\Omega_{\mathrm{sm}}} d \eta d \xi F_{\alpha}(\eta, \xi) \delta\left[\varepsilon-h \nu\left(\eta, \xi^{2}\right)\right],
$$

где $\Omega_{\mathrm{sm}}$ - область переменных мягких мод. Для рассматриваемых гармонических колебаний мягких мод, как обычно, $h \nu\left(\eta, \xi^{2}\right) \simeq h \nu(\eta) \simeq h \nu_{\mathrm{D}} \eta^{1 / 2} \propto k^{1 / 2}$, в значительном большинстве соответствующих в действительности одноямным потенциалам, $V(x) \simeq k x^{2} / 2, k \approx k_{0} \eta \ll k_{0}$, при $9 \xi^{2} / 32<\eta \ll 1$ и $\xi \approx 0$. Тогда из (8) и (10) следует, что

$$
\begin{aligned}
2 h \gamma_{\mathrm{ac}}^{(\alpha)}(\nu) & \approx \frac{\beta^{2}}{M s_{1}^{2}}\left(w g_{h \nu}^{(\alpha)}(h \nu)\right)=C w\left(\frac{h \nu}{w}\right)^{2 \alpha}, \\
g_{h \nu}^{(\alpha)}(h \nu) & \approx 2 \int_{0}^{\infty} d \eta \int_{0}^{(32 \eta / 9)^{1 / 2}} d \xi F_{\alpha}^{0} \eta^{\alpha-1} \delta\left(\varepsilon-h \nu_{\mathrm{D}} \eta^{1 / 2}\right)=Q w^{-1}\left(\frac{\varepsilon}{w}\right)^{2 \alpha} .
\end{aligned}
$$

Здесь безразмерные параметры среды $C=\beta^{2} Q /\left(M s_{1}^{2} w\right)$ и $Q_{\alpha}=q_{0} F_{\alpha}^{0} \eta_{\mathrm{L}}^{\alpha+1 / 2} \equiv Q$ при $q_{0}=4(32 / 9)^{1 / 2} \approx 10$ не зависят от $\alpha \quad(\alpha=1,2)$; это означает, что $F_{1}^{0} \simeq F_{2}^{0} \eta_{\mathrm{L}}$ при $\eta_{\mathrm{L}} \simeq\left(w /\left(M s_{1}^{2}\right)\right)^{1 / 2} \approx 10^{-2}$. Независимость параметров от $\alpha$ имеет место в силу экспериментально найденной универсальности динамических и тепловых свойств стекол при очень низких частотах и температурах, $\nu \lesssim \nu_{\mathrm{m}}=k T_{\mathrm{m}} / h$ и $T \lesssim T_{\mathrm{m}} \approx 1 \mathrm{~K}$, а также в силу отождествления (в теоретической “туннельной" модели [5], [1]) соответствующих возбуждений с туннельными состояниями, для которых асимметрия $\Delta$ и мощность барьера $S$ (при амплитуде туннелирования $\Delta_{0} \propto e^{-S}$ ) подчинены плотности распределения $F(\Delta, S) \approx F_{0}=$ const. В этой аппроксимации концентрация туннельных состояний есть $c_{\mathrm{TS}} \approx F_{0} h \nu_{\mathrm{m}} / w \approx 0.1 F_{0}$, а $F_{0}$ можно найти из сравнения этой оценки с оценкой $c_{\mathrm{TS}} \approx 10^{-5}$, полученной из экспериментальных данных, так 
что $F_{0} \approx F_{\alpha}^{0} \eta_{\mathrm{L}}^{\alpha+1 / 2} / w \approx 10^{-4}, Q \approx q_{0} F_{0} \approx 10^{-3}$ и $C \approx 0.1$ при типичных значениях $\beta \approx 1$ эВ, $M s_{1}^{2} \approx 10$ эВ и $w \approx 10^{-3}$ эВ.

Теперь можно различать два предельных типа ширины $\gamma_{\mathrm{ac}}^{(\alpha)}(\nu)$ и плотности состояний $g_{h \nu}^{(\alpha)}(q)$ :

$$
2 h \gamma_{\mathrm{ac}}^{(\alpha)}(\nu) \approx h \nu\left(\nu / \nu_{\mathrm{eff}}^{(\alpha)}\right)^{2 \alpha-1}, \quad g_{h \nu}^{(\alpha)}(\varepsilon) \approx\left(\varepsilon_{\mathrm{eff}}^{(\alpha)}\right)^{-1}\left(\varepsilon / \varepsilon_{\mathrm{eff}}^{(\alpha)}\right)^{2 \alpha},
$$

где $h \nu_{\mathrm{eff}}^{(\alpha)}=w C^{-1 /(2 \alpha-1)}$, a $\varepsilon_{\mathrm{eff}}^{(\alpha)}=w Q^{-1 /(2 \alpha+1)}, \alpha=1,2$. Как видно из уравнений (1)

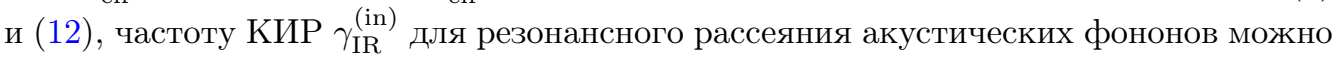
оценить как [13]

$$
h \nu_{\mathrm{IR}}^{(\mathrm{in})} \simeq h \nu_{\mathrm{eff}}^{(\alpha)}=\mu_{\alpha} w \sim h \nu_{0}, \quad \mu_{1} \approx 10, \quad \mu_{2} \approx 3,
$$

так что

$$
\nu_{\mathrm{S \Pi}} \simeq \nu_{\mathrm{IR}}^{(\mathrm{in})} \sim \nu_{0} \sim 1 \mathrm{~T} \mathrm{~T}
$$

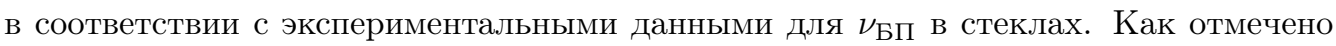
выше, приведенные оценки выполнены для независимых мягких мод в стекле.

Как показано в работе [13] в теории мягких мод для БП, за которым следует ВЧЗ, универсальные дипольные взаимодействия между мягкими модами не меняют существенно форму БП и его частоту $\nu_{\mathrm{Б} \Pi} \simeq \nu_{\mathrm{IR}}^{(\mathrm{in})}$, как и форму спектра ВЧЗ, для полной плотности состояний гармонических колебательных возбуждений $g(\nu)$ и соответствующих $J\left(\nu^{2}\right)=g(\nu) / 2 \nu$ и $g(\nu) / \nu^{2}$. С другой стороны, плотность состояний определяется вещественной частью собственной энергии возбуждений, а ширина возбуждения - мнимой частью. Поскольку эти две части собственной энергии можно связать друг с другом выражениями типа формулы Крамерса-Кронига [21], можно предполагать в данной качественной и верной по порядку величины (но не количественной) теории, что взаимодействия не должны радикально менять приближенные степенные функции и их показатели в (12), описывающие ширину возбуждений.

Это предположение не противоречит теории колебательной плотности состояний гармонических мягких мод $g_{\mathrm{hv}}(\nu)$ [22], с учетом дипольных взаимодействий между мягкими модами в некотором приближении, когда мягкие моды редуцированы к модам эффективных осцилляторных кластеров мягких мод (по-видимому, эффективных осцилляторных пар). Основной результат, полученный в таком приближении, по-видимому, состоит в том, что колебательную плотность состояний гармонических мягких мод $g_{\mathrm{hv}}(\nu)$ при умеренно низких частотах можно аппроксимировать степенной функцией $\left.f(z)\right|_{z \equiv \nu / \nu_{\mathrm{b}}} \propto z^{\varkappa}$, где показатель варьируется от $\varkappa=4$ при $z<1$ до $\varkappa=1$ при $1<z \ll \nu_{\mathrm{D}} / \nu_{\mathrm{b}}$; здесь $\nu_{\mathrm{b}}$ - характеристическая частота, вокруг которой редуцированная плотность состояний $g_{\mathrm{hv}}(\nu) / \nu^{2}$ демонстрирует сравнительно слабый максимум и при этом $J_{\mathrm{hv}}\left(\nu^{2}\right)=g_{\mathrm{hv}}(\nu) / 2 \nu \simeq$ const и $g_{\mathrm{hv}}(\nu) \propto \nu$. Поскольку частота $\nu_{\mathrm{b}}$ количественно близка к частоте $\nu_{\text {БП}}$, определенной явлением КИР для неупругого рассеяния, и интерес представляет частотная зависимость ширины возбуждения при низких частотах $\nu<\nu_{\text {БП }}$, зависимость $2 \gamma_{\text {ас }}(\nu)$ следует находить при $\nu<\nu_{\mathrm{b}}$ и при $\nu \sim \nu_{\mathrm{b}}$ (т.е. точно в области перехода от $\varkappa=4$ для достаточно низких

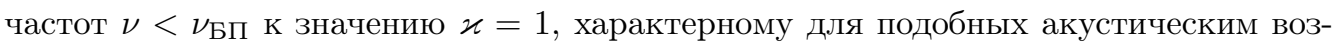
буждениям вблизи БП). Поскольку "промежуточные" значения $\varkappa \approx 2$ могут быть 
приближенной характеристикой этой области перехода, степенную функцию $z^{\varkappa}$ с переменным показателем $\varkappa$ можно аппроксимировать в виде двух непрерывно связанных степенных функций, одной при $\varkappa \approx 2 \alpha=4$ для низких частот и другой с

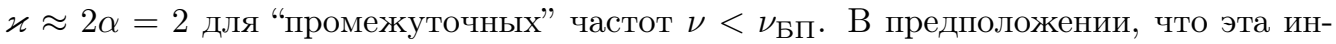
терпретация адекватна, можно видеть, что уравнение (12) справедливо при $\varkappa \approx 2$, т.е. приближенно $2 h \gamma_{\text {ac }}(\nu) \propto \nu^{2}$, при “промежуточных" частотах, что приводит (при определенном условии, см. выше) также к частичному качественному объяснению недавних экспериментальных данных $\mathbf{3}$ из раздела 1. Наконец, оценку характерной

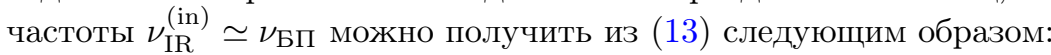

$$
h \nu_{\mathrm{B \Pi}} \simeq h \nu_{\mathrm{IR}}^{(\mathrm{in})} \simeq \mu_{1} w \simeq h \nu_{\mathrm{eff}}^{(\varkappa)} \approx h \nu_{0}, \quad \mu_{1} \approx 10 .
$$

\section{4. ОБСУЖДЕНИЕ}

Формулы (11)-(14), как и соотношение (15) и связанные с ними замечания, могут

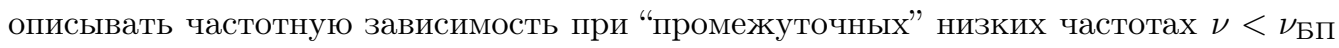
не зависящей от температуры ширины $2 \gamma_{\text {ac }}(\nu)$ акустических фононов, взаимодействующих с гармоническими колебаниями мягких мод в стеклах, демонстрирующих как БП, так и ВЧЗ. Соответствующая полная плотность состояний, например $\left.g(\varepsilon)\right|_{\varepsilon=h \nu}$, одночастичных гармонических колебательных возбуждений определяется почти резонансно взаимодействующими акустическими фононами и колебаниями мягких мод, тогда как БП-возбуждения связаны с КИР для неупругого (резонансного) рассеяния фононов на колебаниях мягких мод. Как представляется, результаты модели мягких мод (см. разделы 1,2 ) дают также качественную (а также верную и по порядку величины) интерпретацию ряда недавних экспериментальных данных по рентгеновскому и нейтронному рассеянию в стеклах [11], включая данные 1, 2 из раздела 1. Добавим, что полученная в теоретической модели мягких мод [1], [13] полная плотность состояний возбуждений гармонических колебаний мягких мод (например, $g(\nu)$ или $g(\nu) / \nu^{2}$ ), описывающая как БП, определяемый явлением КИР для неупругого рассеяния акустических фононов, так и ВЧЗ, выглядит не противоречащей экспериментально найденному экспоненциальному убыванию

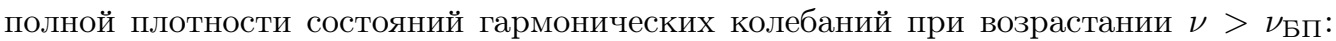

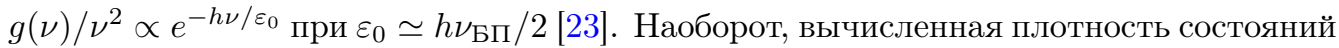
$\left.g_{h \nu}(\varepsilon)\right|_{\varepsilon=h \nu}$ (равенства (11), (12) и (15)) гармонических колебательных возбуждений мягких мод ведет себя иным образом: хотя $g_{h \nu}(\varepsilon) \varepsilon^{-2}$ демонстрирует максимум при

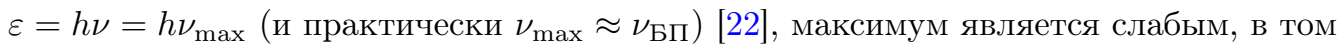
смысле что $g_{h \nu}(\varepsilon) / \varepsilon \approx$ const и $g_{h \nu}(\varepsilon) \propto \varepsilon$ при $\varepsilon \approx h \nu_{\max }$, и не показывает экспоненциального убывания при возрастании $\nu>\nu_{\max }$. Этот факт наряду с другими подчеркивает существенное различие между гармоническими колебательными возбуждениями мягких мод [22] и плохо определенными возбуждениями вблизи БП при $\nu \approx \nu_{\text {БП }} \simeq \nu_{\mathrm{IR}}^{\text {(in) }}$, которые связаны с КИР [13] (сильно неупругое рассеяние акустических фононов на гармонических колебаниях мягких мод и их гибридизация).

Поскольку в спектре ниже и выше щели возбуждения можно рассматривать как распространяющиеся волны, представляется разумным предположить, что неволнообразные щелевые состояния также являются нелокализованными (хотя для это- 
го предположения нет математически строгого доказательства, в настоящее время делается попытка показать, что щелевые состояния скорее похожи на ранее предложенные “диффузоны” [24], которые не описываются как локализованные состояния).

Недавно были предложены две другие теоретические модели (не связанные явно с колебаниями мягких мод), также объясняющие указанные выше экспериментальные данные (см. данные 1-3 из раздела 1). В обеих моделях динамический структурный фактор $S(\bar{q}, \nu)$ был вычислен для гармонических колебательных возбуждений в стеклах с ВЧЗ выше БП. Одна модель [25] основана на теории взаимодействия мод для фазового перехода жидкость-стекло, а также на ее адекватной экстраполяции на стекольную фазу и на симуляциях в модифицированной структурной модели твердых сфер для стекла. Не зависящая от температуры ширина акустических фо-

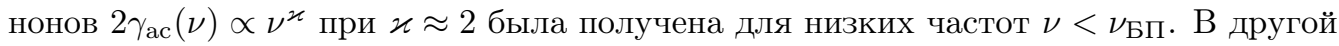
модели [26], исследующей спектры гармонических колебаний в стеклах, некоторые результаты экспериментов по рентгеновскому и нейтронному рассеянию в таких аморфных материалах интерпретировались в рамках теории евклидовых случайных матриц. Это позволяет учесть топологический беспорядок, который влияет на спектры колебаний в стекле. Этот подход основан на процедуре частичного суммирования разложения теории возмущений по обратной (высокой) плотности частиц, что приводит к точному аналитическому выражению динамического структурного фактора, которое можно подходящим образом экстраполировать на диапазон плотностей, типичных для реальных стекол. Представляется интересным явно выявить природу дополнительных, недебаевских, гармонических колебательных возбуждений (если они отличаются от гармонических колебательных возбуждений мягких мод) в этих двух моделях.

Уместно добавить, что для стекол, не обладающих ВЧЗ выше БП, имеются два различных типа (не связанных с мягкими модами) моделей происхождения БП и частотной зависимости ширины акустического фонона $2 \gamma_{\text {ac }}(\nu)$ (не зависящей от тем-

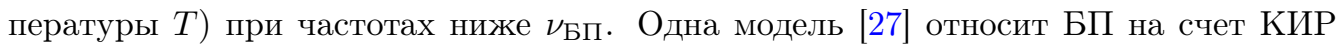
для упругого рассеяния акустических фононов на статическом беспорядке и приводит к частотной зависимости $2 \gamma_{\text {ac }}(\nu) \propto \nu^{\varkappa}$ при типичных для рэлеевского рассеяния значениях $\varkappa \approx 4$. В другой модели [11], [28] предполагается, что важен только статический беспорядок, который приводит к микроскопическим случайным пространственным флуктуациям поперечных констант упругости (модуля сдвига). Тогда плотность состояний избыточных, недебаевских, возбуждений $\Delta g_{\text {exс }}(\nu)$ и связанный с ней БП возникают из зоны случайных поперечных акустических колебательных состояний. В этой модели (не связанной с КИР для упругого рассеяния акустических фононов) экспериментально наблюдаемую частотную зависимость

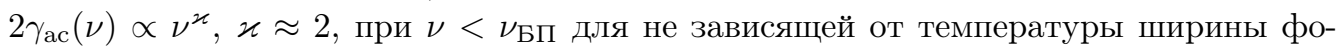
нона можно, по-видимому, получить путем надлежащих вычислений. В этой связи представляется интересным найти причину различия зависимостей $\gamma_{\text {ac }}(\nu) \propto \nu$ при $\varkappa \approx 4$ и при $\varkappa \approx 2$, а также разной роли поперечных акустических фононов в этих двух моделях. 


\section{Список литературы}

[1] M. I. Klinger, Phys. Rep., 165 (1988), 275; Philos. Mag. B, 81 (2001), 1273.

[2] M. I. Klinger, A. M. Kosevich, Phys. Lett. A, 280 (2001), 365.

[3] W. A. Phillips (ed.), Amorphous Solids: Low-temperature Properties, Springer, Berlin, 1981.

[4] G. Ruocco, F. Sette, J. Phys. Condens. Matter, 13:41 (2001), 9141; A. Matic, L. Boerjesson, G. Ruocco, C. Masciovecchio, A. Mermet, F. Sette, R. Verbeni, Europhys. Lett., 54 (2001), 77.

[5] P. W. Anderson, B. I. Halperin, C. M. Varma, Philos. Mag., 25 (1972), 1; W. A. Phillips, J. Low Temp. Phys., 7 (1972), 351.

[6] М.И. Клингер, "Небольцмановские типы электронного переноса в неметаллических твердых телах", Проблемы современной физики, ред. А.П. Александров, Наука, Л., 1980, 293.

[7] M. I. Klinger, V. G. Karpov, Solid State Commun., 37 (1981), 975; В. Г. Карпов, М. И. Клингер, Ф.Н.Игнатьев, ЖЭТФ, 84 (1983), 760.

[8] U. Buchenau, M. Ramos, "Soft-potential model", Chapter 9, Tunneling Systems in Amorphous and Crystalline Solids, ed. P. Esquinazi, Springer, Berlin, 1998, 527.

[9] R. Shuker, R. W. Gamon, Phys. Rev. Lett., 25 (1970), 222.

[10] C. A. Angell, J. Phys. Condens. Matter, 12:29 (2000), 6463.

[11] W. Schirmacher, G. Ruocco, T. Scopigno, Phys. Rev. Lett., 98 (2007), 025501.

[12] M. I. Klinger, A. M. Kosevich, Phys. Lett. A, 295 (2002), 311.

[13] M. I. Klinger, L. Vatova, Phys. Rev. B, 72 (2005), 134206.

[14] A. F. Ioffe, A. R. Regel, Prog. Semicond., 4 (1960), 237.

[15] A. L. Burin, D. Natelson, D. D. Osheroff, Yu. Kagan, "Interactions between tunneling defects in amorphous solids", Chapter 5, Tunneling Systems in Amorphous and Crystalline Solids, ed. P. Esquinazi, Springer, Berlin, 1998, 223.

[16] R. Brout, W. Visscher, Phys. Rev. Lett., 9 (1962), 54; Ю. Коган, Я. А. Иосильевский, ЖЭТФ, 42 (1962), 259.

[17] И. М. Лифшиц, С.А. Гредескул, Л.А. Пастур, Введение в теорию неупорядоченных систем, Наука, М., 1982.

[18] P. W. Anderson, Phys. Rev., 109 (1958), 1492.

[19] M. I. Klinger, V. Halpern, Phys. Lett. A, 313 (2003), 448.

[20] U. Buchenau, Yu.M. Galperin, V. L. Gurevich, D. A. Parshin, M. A. Ramos, H. R. Schober, Phys. Rev. B, 46 (1992), 2798.

[21] H. A. Kramers, Atti Congr. Inter. Fis. (Como), 2 (1927), 545; R. Kronig, J. Opt Soc. Amer., 12 (1926), 547.

[22] V. L. Gurevich, D. A. Parshin, H. R. Schober, Phys. Rev. B, 67 (2003), 094203.

[23] A. I. Chumakov, I. Sergueev, U. van Bürck, W. Schirmacher, T. Asthalter, R. Rüffer, O. Leupold, W. Petry, Phys. Rev. Lett., 92 (2004), 245508.

[24] J. L. Feldman, P. B. Allen, S. R. Bickham, Phys. Rev. B, 59 (1999), 3551.

[25] W. Goetze, M. R. Mayr, Phys. Rev. E, 61 (2000), 587.

[26] T.S. Grigera, V. Martin-Mayor, G. Parisi, P. Verrocchio, Philos. Mag., 82 (2002), 637.

[27] S. N. Taraskin, S. R. Elliott, J. Phys. Condens. Matter, 14:12 (2002), 3143; S. N. Taraskin, J. J. Ludlam, G. Natarajan, S. R. Elliott, Philos. Mag. B, 82 (2002), 197.

[28] W. Schirmacher, Europhys. Lett., 73 (2006), 892. 